\title{
Management of the Tolerance to Bolting for Spinach (Spinacia Oleracea L.): Retrospect and Prospects
}

\author{
Guoliang Li", Qing Meng*, Zhiyuan Liu, Zhaosheng Xu, Helong Zhang and Wei Qian* \\ Institute of Vegetables and Flowers, Chinese Academy of Agricultural Sciences, China \\ ${ }^{\#}$ These two authors contributed equally to this work. \\ *Corresponding author: Wei Qian, Institute of Vegetables and Flowers, Chinese Academy of Agricultural Sciences, 12\# Zhong \\ Guan Cun South Street, Haidian District, Beijing 100081, China
}

\section{ARTICLE INFO}

Received: 慧 May 25, 2020

Published: 蔧 June 03, 2020

Citation: Guoliang Li, Qing Meng, Zhiyuan Liu, Zhaosheng Xu, Helong Zhang, Wei Qian. Management of the Tolerance to Bolting for Spinach (Spinacia Oleracea L.): Retrospect and Prospects. Biomed J Sci \& Tech Res 28(1)-2020. BJSTR. MS.ID.004595.

Keywords: Spinach; Bolting Tolerance; Photoperiod; Gibberellic Acid; Bolting Breeding

\section{ABSTRACT}

Spinach (Spinacia oleracea L.) is an important and nutritious green leafy vegetable, and there are rich carotenoids, folate, vitamin C, calcium and iron in spinach. The yields of spinach were approach 28 million tons in 2017 all over the world (FAO). However, the earlier bolting is one of the important limiting factors affecting the quality and yield in spinach, and it is an instant task to preventing the earlier bolting and creating the bolting tolerance germplasm resources in spinach breeding. The evaluation criteria is essential to determine whether it has entered the reproductive stage and to judge the timing of harvesting or breeding, but the evaluation criteria for the bolting trait in spinach had not been established yet. Bolting is controlled by some endogenous and environmental factors. The bolting variation provided extremely rich resources in spinach, and the abiotic stresses could induce bolting and the biochemical regulations such as endogenous and exogenous hormones could also influence the bolting mechanism.

A diverse range of endogenous and environmental signals regulates bolting, and these signals are integrated into a single decision. The study on bolting characteristics was summarized in terms of factors influencing bolting and inheritance of bolting characteristics, to serve as reference for the further study in spinach. Because of its sensitivity to photoperiod, long-day exposure induces bolting in spinach, which is not good for the year-round production in spinach. Manipulating the day length and temperature during the growing process of spinach can prevent bolting. The previous studies showed that bolting tolerance is associated with an expression of an integrative gene network involved in environmental perception, hormone signalling, and flowering in the shoot apical meristem. However, the genotypic tolerance to bolting, particularly important for breeders, is still poorly understood in spinach. This review on bolting trait may lay the foundation for bolting-resistant breeding in spinach.

\section{Introduction}

Spinach is a diploid $(2 \mathrm{n}=12)$ that is native to the mid-east probably Iran Ryder [1], and it was first mentioned in China around 600 A.D. as the herb of Persia Nonnicke [2]. It is a member of the family Amaranthaceae, which also contains sugar beet, chard and quinoa as well as some weeds like lamb's-quarters Morelock, et al. [3]. Spinach is an important and nutritious green leafy vegetable, and there were rich carotenoids, folate, vitamin $\mathrm{C}$, calcium and iron in spinach Dicoteau [4]. Besides, spinach is a good source of antioxidants and has one of the highest ORAC (oxygen radical absorbance capacity) values of any vegetable Prior [5]. Normally, spinach was used as a cooked vegetable or a salad or cooked meat and vegetable dishes or as an ingredient in fresh or frozen spinach or canned spinach Morelock, et al. [3]. Spinach were popular all around the world, and the production increased to $\sim 28$ million tons in 2017, including 26 million tons accounting for 92\% in China (http://www.fao.org/faostat/zh/?\#data/QC). Spinach is a longday plant, which is harvested when its rostte leaves are nearly fully expanded at its vegetative growth stage Hartmann et al. [6].

The onset and development of the reproductive phase involve the distinct and sequential processes of bolting followed 
by flowering Mutasa-Göttgens, et al. [7]. After transition to the reproductive stage with flowering and bolting, spinach loses its value as an agricultural product, becoming bitter and hard Abe, et al. [8]. Bolting is an important agronomy trait to consider in relation to developing spinach cultivars for year-round production, because of its sensitivity to photoperiod Chun, et al. [9]. Because some commercially grown spinach is cut multiple times Morelock, et al. [3], overwintered spinach that is susceptible to bolting in the spring reduces the number of harvests that may be taken and therefore reduces overall yield. To prevent this phenomenon, the appropriate cultivars are selected for cropping. This review would summarize the late-bolting research progress and the factors that influencing bolting in spinach, and may lay the foundation for the molecular design late-bolting breeding in spinach.

\section{Evaluation Criteria for the Bolting Trait}

As a quantitative trait, bolting is influenced and regulated by many environmental and endogenous factors (Gómez-Mena et al., 2001), which makes it hard to evaluate. At present, the identification of morphological index of bolting is the common evaluation criteria for the bolting traits and it mainly depends on subjective observation, measurement and statistics. In previous study, bolting rate was estimated as percentage of plants bolted per replication, that is: $0=$ none; $1=1 \%$ to $20 \% ; 2=21 \%$ to $40 \%$; $3=41 \%$ to $60 \% ; 4=61 \%$ to $80 \% ; 5=81 \%$ to $100 \%$ Goreta, et al. [10]. In addition, an accession was deemed 'early bolting', if any plant started stem elongation earlier than $60 \mathrm{~d}$ after planting, 'intermediate' if bolting between 60 and $70 \mathrm{~d}$, and 'late bolting' after the $70 \mathrm{~d}$ Chitwood, et al. [11]. Xu, et al. [12] bolting time was monitored daily and determined as the number of days from planting to the first elongation of the floral stem observed in at least half of the used plants for each accession. There were few studies on the late-bolting in spinach, and the standard of the late-bolting phenotype investigation has not been established yet.

In the previous study, our team tried a set of standards, formulating the standards as follows: bolting stage (BS) is confirmed firstly, which is the days from the planting day to the day of the length of $50 \%$ individuals elongate stem $\geq 5 \mathrm{~cm}$. According to the elongate stem length (ESL) in bolting stage, the spinach individuals can be divided into five bolting levels (BL) (BL 0: ESL $=0 \mathrm{~cm}$; BL 1: $0 \mathrm{~cm}<\mathrm{ESL} \leq 5 \mathrm{~cm}$; BL 2: $5 \mathrm{~cm}<\mathrm{ESL} \leq 10 \mathrm{~cm}$; BL $3: 10 \mathrm{~cm}<\mathrm{ESL} \leq$ $15 \mathrm{~cm}$; BL $4: 15 \mathrm{~cm}<\mathrm{ESL} \leq 20 \mathrm{~cm}$; BL5 : ESL $020 \mathrm{~cm}$ ). In addition, the bolting index (BI) could obtain, that is, $\mathrm{BI}=\sum[$ (BL grade $\times$ Number of plants of every BL) / (Number of total plants $\times 5$ )] $\times 100 \%$. The BI values were used to evaluate the spinach populations, "high tolerance (HT)' if $0<\mathrm{BI}<0.2$; 'tolerance (T)' if $0.2 \leq \mathrm{BI}<0.4$; 'middle tolerance (MT)' if $0.4 \leq \mathrm{BI}<0.6$; 'low tolerance (LT)' if $0.6 \leq \mathrm{BI}<$ 0.8 ; 'early (E)' if $0.8 \leq \mathrm{BI}<1.0$ (unpublished data). The standards above were used to evaluate the BC1 and F2 populations from the parents 12S3 (late-bolting) and 12S4 (easy-bolting), and the major late-bolting QTL was mapped in the range of 48.12 to $49.2 \mathrm{Mb}$ in Chromosome 1 (unpublished data).

\section{Factors that Influencing Bolting}

As every plant individual with a short commodity value growth period, spinach no longer has a good flavor when it begins to bolt. Therefore, it is important to find a method of inhibiting or delaying bolting Borkowski, et al. [13]. A wide range of genetic and environmental factors are involved in this transition, and signals from those factors are integrated into a network (Song et al. 2015). The factors that affect bolting in spinach include genotypes, abiotic stresses, biochemical regulation and other internal or external factors.

Different Genotypes in Spinach: The bolting variation in natural spinach provides extremely rich resources for breeding. Resistance to bolting among different cultivars has been known for a long time, which has the character of longer growth periods Webb, et al. [14]. It has been shown that the thorn varieties have a strong bolting tolerance. Generally, oriental cultivars that Japanese and other Asian customers prefer bolt easy Chun, et al. [9]. Low bolting rate was found in the cultivars 'Fidalgo', 'Springfield', and 'Springer' Goreta, et al. [10], which will improve the efficiency of breeding and producing in spinach.

Abiotic Stresses: Abiotic stress factors such as chilling, drought, and strong light also induce bolting in spinach. Bolting time was negatively correlated with irradiance, which led to oxidative stress and might induce earlier bolting in Arabidopsis thaliana (Moharekar et al., 2007). Bolting is not enhanced if seed is exposed to low temperatures during development on the motherplant Wiebe [15]. But bolting rate increase if cold exposure of growing plants is followed by higher temperatures and long days (Yamaguchi, 1983). Chun et al. (1999) showed that manipulating the day length and temperature during transplant production can prevent bolting by using artificial light. Spinach is a long-day plant, a long photoperiod causes it to bolt and flower, Kim, et al. [16] reported that spinach plants began to sense the photoperiod during transplant production, and that flower bud development during this period was enhanced under a longer photoperiod. The photoperiod during transplant production has a significant effect on the percentage of bolting plant, and the delay of bolting that occurs when the photoperiod is reduced Chun, et al. [17]. And its flowerstalk length increased with increasing photoperiod (e.g., at 14 DAT, from $15 \mathrm{~mm}$ at the shorter photoperiods to $80 \mathrm{~mm}$ at 16 hours/ day) Chun, et al. [17]. Before transplanting, short photoperiod treatments can retard bolting while long-day exposure induces bolting Goreta, et al. [10].

Biochemical Regulation: The tip-shoot, as a hypothermic sensing part, is the key part of vernalization from vegetative growth stage to reproductive growth stage in spinach. The information of bolting could be obtained by detecting the biochemical substance in the critical period of flower bud differentiation. In Brassicaceae, different varieties showed consistent regularity to the stimulation of phytohormones in bolting, that is, the varieties with early flower 
bud differentiation and early bolting were sensitive to the signal stimulation Yu, et al. [18], which would provide the reference frame to our study. Phytohormones, such as gibberellins and auxin, have been documented to regulate flowering transition and organ size (Davies, 2003). Gibberellic acid (GA) can regulate various ways of plant growth and development such as seed germination, leaf expansion, stem elongation and flower development through promoting cell division and cell elongation Richards, et al. [19]. The regulation of GA metabolism plays an important role in controlling bolting related to day length in spinach Zeevaart, et al. [20]. Besides, GA has been shown to promote flowering of Arabidopsis thaliana by activating the LEAFY promoter, and crosstalk with photoperiod and vernalization pathways $\mathrm{Yu}$, et al. [21]. Among chemical compounds, maleic hydrazide $(\mathrm{MH})$ is one which inhibits plant growth by interfering with cell division. $\mathrm{MH}$ and its commercial preparation 'Antyrost' were found to have a strong inhibition on bolting Greulach, et al. [23,24].

\section{Genetic Analysis of the Late-Bolting Trait}

All the studies on the inheritance all over the world showed that the bolting trait is regulated by multigene, and early-bolting gene is incomplete dominant to late-bolting gene $\mathrm{Yu}$, et al. [18]. In order to develop such spinach cultivars, it is necessary to understand flowering and bolting mechanisms of spinach at the genetic level. Genetic and molecular studies have revealed that many genes are involved and can be assigned to distinct regulatory pathways including photoperiod, vernalization, gibberellin, autonomous, ambient temperature, and age pathways Fornara, et al. [24]). These pathways converge to regulate a set of 'floral integrator' genes that integrate the outputs of the various pathways and directly activate the identity genes of the floral meristem Putterill, et al. [25]. These integrator genes include SUPPRESSOR OF OVEREXPRESSION OF CONSTANS 1 (SOC1), FLOWERING LOCUS T (FT), TWIN SISTER OF FT (TSF), FLOWERING LOCUS C (FLC) ?EAFY (LFY) and so on Fornara, et al. [24,25].

In Arabidopsis, most key flowering genes have been identified and functionally characterized. SOC1 encodes a MADS-box protein that integrates multiple flowering signals derived from photoperiod, temperature, hormone and age-related pathways (Lee, 2010). Interestingly, it has been discovered that many homologous genes of Arabidopsis regulate flowering in other species (Song et al., 2015). Regulations of spinach flowering and bolting by photoperiod and gibberellin have been reported Zeevaart, et al. [20,26]; Lee \& Zeevaart, 2002, Lee, et al. [27,28]. To understand spinach flowering and bolting mechanisms at a genetic level, one CONSTANS-like (COL) and two FLOWERING LOCUS T (FT) homologs were isolated and characterized, which are known to be key factor of the flowering pathway in many plants Abe, et al. [8]. Detecting the mechanisms to regulate pathways in control of spinach bolting could promote the related genetic discovering. In recent years, the molecular markers with bolting have been developing.
For assessing genetic diversity within accessions, $\mathrm{Hu}$, et al. [29] analyzed genetic variability among 38 germplasm accessions and 10 commercial hybrids of spinach by using target region amplification polymorphism (TRAP) markers. In Kuwahara's study, the simple sequence repeat (SSR) data were used to study the population structure and genetic diversity of the 48 spinach accessions Kuwahara, et al. [30]. A total of 288 spinach accessions from United States Department of Agriculture (USDA) were used as the association panel to discovered the single-nucleotide polymorphisms (SNPs) through genotyping by sequencing (GBS), and three SNP markers, AYZV02001321_398,AYZV02041012_1060, and AYZV02118171_95 were identified to be associated with bolting Chitwood, et al. [11]. The draft genome sequence of spinach was reported, and a number of known markers and quantitative trait loci (QTLs) associated with bolting were obtained in the region from 44.7 to $50.5 \mathrm{Mb}$ of Chromosome $2 \mathrm{Xu}$, et al. [12]. In addition, spinach and sugar beet were both belong to the Amaranthaceae family. In sugar beet, 40 genes associated with bolting were respectively obtained by amplified fragment length polymorphism (AFLP) and iTRAQ technology Liang, et al. [31]. The studies about molecular markers of bolting on other Amaranthaceae family specials may be laid the foundation for the further study in spinach.

\section{Breeding of the Late-Bolting Varieties}

Understanding the molecular genetic mechanism of the bolting could provide evidence for their application in breeding. There were the gaps between the bolting research and the spinach crop, and much work should been done to develop and analyze the bolting genes in spinach. The bolting genes could be applied in breeding in many ways, such as transformation of the bolting genes, molecular marker assisted selection (MAS), late-bolting genes pyramiding, and so on. It is our goal to take full advantage of the bolting genetic information for breeding.

\section{Prospects of the Bolting Trait in Spinach}

Spinach cultivation has a long history, wide geographical distribution and rich germplasm resources, which provide the basis of plant breeding and genetic research. There were a total of 1,938 germplasm resources of spinach that have been counted in the world Kik [32]). The germplasm resources of spinach are widely used as experimental materials for the study of plant molecular biology and photosynthetic mechanism. Besides the bolting stage (BS) and the elongate stem length (ESL) that we mentioned above, Days to Flowering (DTF), Days to Bolting (DTB) and Number of leaves in flowering (NLF) are also used to identify bolting index. There is no uniform international standard for the bolting trait. At present, the identification of morphological index of bolting is the common evaluation criteria for the bolting traits, but this is highly subjective. In Chinese cabbage, the varieties with early flower bud differentiation and early bolting were sensitive to the signal stimulation $\mathrm{Yu}$, et al. [18]. The content of CTK and three polyamines (spermidine putamine spermidine) of late bolting varieties 
were lower than that of early bolting varieties Cheng, et al. [33] These physiological changes during the bolting could be used as a reference for the selection of late-bolting varieties.

The combination of physiological indexes and morphological index would be helpful to the study of the bolting trait. Genetic variation among spinach and resistance to bolting among different cultivars for bolting has been documented for many years Goreta, et al. [10], and therefore late-bolting cultivars could be developed through breeding efforts Brandenberger, et al. [34]. Areas with mild climate conditions are suitable for growing winter spinach, and successful production depends on choosing late bolting cultivars. For example, three cultivars (Fidalgo, Springfield, and Springer) showed late-bolting traits from a total of 18 cultivars during 8 years in the Winter Garden region of Texas Goreta, et al. [10]. For further study, we can detect the different varieties of spinach from mild climate condition in winter to take full advantage of the bolting genetic information for breeding. Bolting is regulated by seasonal cues to help ensure reproductive success, synchronous flowering in agriculture, and maximal yield.

So it is very important to understand the mechanisms of reproductive phase (flowering and bolting) transition in spinach. Change the seasonal timing of flowering could allow the production of novel varieties adapted to local climatic and environmental conditions Jung, et al. [35]. Breeding targets for the control of bolting in cultivating must meet the dual, but opposing, needs for high yielding crops and seed production Mutasa-Göttgens, et al. [7]. Late-bolting spinach is preferred to increase market value, while early-bolting spinach is beneficial for seed production. As a longday plant, the photoperiod has a great limitation on the year-round production of spinach. Early-bolting or late-bolting is both good for grown spinach if it lacks the sensitivity to photoperiod. So further analysis need to understand both the early-bolting and late-bolting about the photoperiod in spinach Chun, et al. [9]. Arabidopsis thaliana is a model facultative long-day (LD) plant Jung, et al. [35]. CONSTANS (CO) plays a central role in the photoperiod pathway in Arabidopsis, and the CO transcription activates transcription of FT and TSF transcription only during long days Fornara, et al. [24]) CO-mutant was insensitive to the day length (Re'dei, 1962).

In genotypes where $\mathrm{CO}$ transcription shifts, $\mathrm{CO}$ activation is no longer restricted to long days but also occurs during short days, leading to earlier FT transcription and flowering under short-day conditions Fornara, et al. [24]. In the next study, CO-mutant or other gene involved in the photoperiod pathway can be used for creating new germplasm to achieve anniversary production in spinach. The flowering genes in Arabidopsis have been well studied, making Arabidopsis a viable reference species for comparative genomic studies. To date, knowledge of the spinach genome is limited and few reports have been published on the use of molecular markers in spinach Khattak, et al. [36]. Molecular assisted selection (MAS) could be further used to select specific genes alleles in spinach breeding. Transgenic approaches are also an obvious and convenient option for crop improvement, and are made easier by the hybrid nature. The use of inducible transgenic targets enabled bolting and flowering to be activated only in the specified lines Mutasa-Göttgens, et al. [7]. To detect the genetic mechanism and regulatory genes of bolting traits by combining biotechnology with conventional breeding will be an important means for future research on bolting breeding of spinach [37-42].

\section{Acknowledgment}

This work was performed at the Key Laboratory of Agriculture, Beijing, China, and was supported by Natural Science Foundation of China (31872102), the Chinese Academy of Agricultural Sciences Innovation Project (CAAS-ASTIP-IVFCAAS), the Key Laboratory of Biology and Genetic Improvement of Horticultural Crops, Ministry of Agriculture, Beijing, China.

\section{Author Contributions}

WQ and GL designed the study. GL and QM wrote the manuscript. ZL and HZ made the revision of the manuscript. WQ, GL, QM, ZL, and ZX approved the final version of the manuscript.

\section{Ethical Standards}

The authors declare that this study complies with the current laws of the countries in which the experiments performed.

\section{Conflict of Interest Statement}

We declare that we do not have any commercial or associative interest that represents a conflict of interest in connection with the work submitted.

\section{References}

1. Ryder EJ (1979) Leafy salad vegetables. AVI. pp. 195-227.

2. Nonnicke IL (1989) Vegetable Production Van Nostrand Runhold. pp. 476-484.

3. Morelock T, Correll J (2008) Vegetables I. In: Prohens J, Nuez F (Eds.) Spinger, pp. 189-218.

4. Dicoteau DR (2000) Vegetable Crops. Prentice Hall. pp. 221-237.

5. Prior RL (2003) Spinach as a source of antioxidant phytochemicals with potential health Effects.2003. National Spinach Conference. p. 20-21.

6. Hartmann HT, Kofranek AM, Rubatzky VE, Flocker WJ (1988) Plant science PrenticeHall Englewood Cliffs N. J.

7. Mutasa Göttgens ES, Aming Q Wenying Z, Schulze Buxloh G, Andrea J, et al. (2010) Bolting and flowering control in sugar beet: relationships and effects of gibberellin the bolting gene $B$ and vernalization. AoB Plants.

8. Abe E, Fujino K, Masuda K, Yamaguchi Y (2014) Isolation and Expression Profiling of a CONSTANS-Like Gene and Two FLOWERING LOCUS T-Like Genes from Spinacia oleracea L. American Journal of Plant Sciences 5(26): 4018-4028.

9. Chun C, Watanabe A, Kozai T, Kim HH, Fuse J (2000) Bolting and growth of Spinacia oleracea L. can be altered by modifying the photoperiod during transplant production. HortScience 35(4): 624-626.

10. Goreta S, Leskovar DI (2006) Screening spinach cultivars for white rust resistance and bolting. HortTechnology 16(1): 162-166.

11. Chitwood J, Shi A, Mou B, Evans M, Clark J, et al. (2016) Population structure and association analysis of bolting plant height and leaf erectness in spinach. HortScience 51(5): 481-486. 
12. Xu C, Jiao C, Sun H, Cai X, Wang X, et al. (2017) Draft genome of spinach and transcriptome diversity of 120 Spinacia accessions. Nat Commun.

13. Borkowski J, Jankiewicz LS (1979) Inhibition of spinach bolting by growth regulators. Acta Agrobotanica 32(2): 233-238.

14. Webb RE, Thomas CE (1976) Development of F1 spinach hybrids. HortScience 11: 546

15. Wiebe HJ (1989) Effects of low temperature during seed development on the mother plant on subsequent bolting of chicory lettuce and spinach. Scientia horticulturae 38(3-4): 223-229.

16. Kim HH, Chun C, Kozai T, Fuse J (2000) The potential use of photoperiod during transplant production under artificial lighting conditions on floral development and bolting using spinach as a model. HortScience 35(1): 43-45.

17. Chun C, Tominaga M, Kozai T (2001) Floral development and bolting of spinach as affected by photoperiod and integrated photosynthetic photon flux during transplant production. HortScience 36(5): 889-892.

18. Yu YJ, Zhang FL, Zhao XY, Zhang DS, He J, et al. (2005) The inheritance of late-bolting in cross of Chinese cabbage $\times$ Chinese cabbage and the intraspecific crosses of Paktroi $\times$ Chinese cabbage and turnip $\times$ Chinese cabbage. Acta Agricultuae Boreali-Sinica 20(3): 17-21.

19. Richards DE, King KE, Ait Ali T, Harberd NP (2001) How gibberelling regulates plant growing and development: a molecular genetic analysis of gibberellin signaling. Annual review of plant physiology and plant molecular biology 52: 67-88

20. Zeevaart JA (1971) Effects of photoperiod on growth rate and endogenous gibberellins in the long-day rosette plant spinach. Plant Physiology 47(6): 821-827.

21. Yu H, Ito T, Zhao Y, Peng J, Kumar P, et al. (2004) Floralhomeotic genes are targets of ibberellins signaling in flower development. Proceedings of the National Academy of Sciences of the United States of America 101(20): 7827-7832.

22. Greulach VA, Atchison E (1953) Inhibition of mitosis in bean buds by maleic hydrazide. Botanical Gazette 114(4): 478-479.

23. Greulach VA, Haesloop JG (1954) Some effects of maleic hydrazide on internode elongation cell enlargement and stem anatomy. American Journal of Botany. p. 44-50

24. Fornara F, Montaigu A, Coupland G (2010) SnapShot: Control of flowering in Arabidopsis. Cell 141(3): 550.

25. Putterill J, Laurie R, Macknight R (2004) It's time to flower: the genetic control of flowering time. Bioessays 26(4): 363-373.

26. Wu K, Li L, Gage DA, Zeevaart JA (1996) Molecular cloning and photoperiod-regulated expression of gibberellin 20-oxidase from the long-day plant spinach. Plant Physiology 110(2): 547-554

27. Lee DJ, Zeevaart JA (2005) Molecular cloning of GA 2-oxidase3 from spinach and its ectopic expression in Nicotiana sylvestris. Plant Physiology 138(1): 243-254.

ISSN: 2574-1241

DOI: 10.26717/BJSTR.2020.28.004595

Wei Qian. Biomed J Sci \& Tech Res

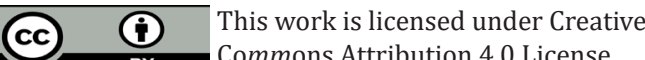

Submission Link: https://biomedres.us/submit-manuscript.php
28. Lee DJ, Zeevaart JA (2007) Regulation of gibberellin 20-oxidase1 expression in spinach by photoperiod. Planta 226(1): 35-44.

29. Hu J, Mou B, Vick BA (2007) Genetic diversity of 38 spinach (Spinacia oleracea L.): germplasm accessions and 10 commercial hybrids assessed by TRAP markers. Genetic Resources and Crop Evolution 54(8): 16671674.

30. Kuwahara K, Suzuki R, Ito Y, Mikami T, Onodera Y (2014) An analysis of genetic differentiation and geographical variation of spinach germplasm using SSR markers. Plant Genetic Resources 12(02): 185-190.

31. Liang N, Cheng D, Liu Q Cui J, Luo C (2018) Difference of proteomics vernalization induced in bolting and flowering transitions of Beta vulgaris. Plant physiology and biochemistry 123: 222-232.

32. KIK C (2013) Two collecting expeditions to the centres of biodiversity of spinach. Proceedings of 2013 International Spinach Conference. Guangzhou: Chinese Horticultural Society.

33. Cheng F, Zhang SN, Sun CH (1999) Morphological and physiological indexes of spring Chinese cabbage cultivars breeding. Journal of nanjing agricultural university 26(2): 120-122.

34. Brandenberger L, Wells LK, Haigh MM (2004) Yield and quality of spinach cultivars for spring production in Oklahoma. HortTechnology 14(4): 602-605.

35. Jung C, Muller AE (2009) Flowering time control and applications in plant breeding. Trends in Plant Science 14(10): 563-573.

36. Khattak J, Torp AM, Andersen SB (2006) A genetic linkage map of Spinacia oleracea and localization of a sex determination locus. Euphytica 148(3): 311-318.

37. Dong J (2019) Collection preservation and genetic diversity analysis of Spinach germplasm resources Changchun: Jilin Agricultural University.

38. Komeda Y (2004) Genetic regulation of time to flower in Arabidopsis thaliana. Annual review of plant biology 55: 521-535.

39. Nie S, Li C, Xu L, Wang Y, Huang D, et al. (2016) Denovo transcriptome analysis in radish (Raphanus sativus L.): and identificationof critical genes involved in bolting and flowering. Genomics.

40. Parcy F (2005) Flowering: a time for integration. The International journal of developmental biology 49(5-6): 585-593.

41. Sadeghian SY, Johansson E (1993) Genetic study of bolting and stem length in sugar beet (Beta vulgaris L.): using a factorial cross design. Euphytica 65: 177-185.

42. Thomas TH, Rubatzky VE, Yamaguchi M (2000) World Vegetables: Principles Production and Nutritive Values. Plant Growth Regulation $30(3): 276-276$

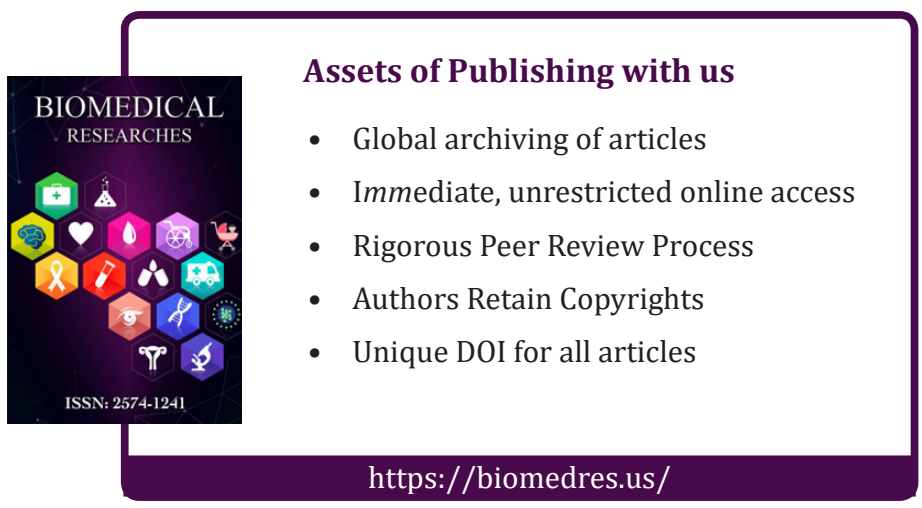

Copyright@ Wei Qian | Biomed J Sci \& Tech Res | BJSTR. MS.ID.004595. 